\section{Candida Cholecystitis as an Unusual Complication of Endoscopic Retrograde Cholangiography}

A 65-year-old man with well-controlled diabetes was admitted with transient diarrhea. The stool culture was positive for Candida albicans at admission, but soon became negative. He underwent endoscopic retrograde cholangiography (ERC) for a suspicion of gallstones with mild common bile duct dilation. The cholangiogram revealed gallstones and intact biliary tracts. Five to seven days later, he developed low-grade fever and pain in the right upper quadrant. The laboratory data were consistent with cholestasis with inflammation, and ultrasonography showed acute cholecystitis. The jaundice and inflammation persisted in spite of antibiotic treatment. He was then treated with endoscopic nasobiliary drainage (ENBD) and percutaneous transhepatic cholecystic drainage (PTCD) (Figure 1), since the marked jaundice and inflammation precluded surgical treatment. A diagnosis of Candi$d a$ cholecystitis was made preoperatively on the basis of positive bile cultures of Candida albicans obtained at PTCD. Although his jaundice and cholecystitis were ameliorated by drainage and lavage with fluconazole via ENBD and PTCD in combination with systemic administration of this agent, it was observed that the candidal infection had spread to the subphrenic space and pleural cavity. He eventually recovered after surgical pleural drainage and lavage with fluconazole, and cholecystectomy. Surgery revealed gallstones with a thickened wall, and a turbid pleural effusion and fibrinous adhesions. The histological examination of the gallbladder showed infiltration of the wall by Candida albicans (Figure 2).

The incidence and importance of Candida infections have increased in immunocompromised patients (1). Although the involvement of almost every organ system by Candida has been reported (2), fungal cholecystitis is rarely described $(3,4)$ or diagnosed preoperatively (5). The onset of cholecystitis several days after ERC in our patient suggests that he most likely developed an ascending retrograde infection of the gallbladder from a gut colonized by Candida albicans. Another possible 


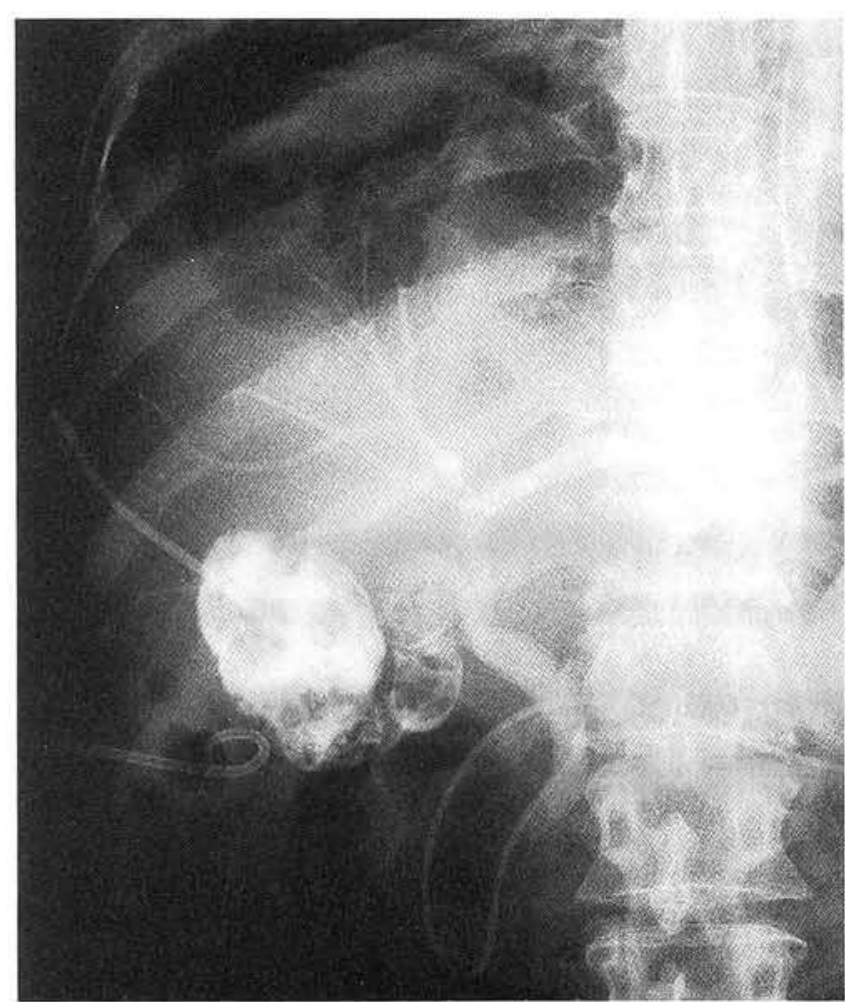

Figure 1: The patient's biliary system is drained and lavaged via PTCD and ENBD. Stones are present only in the gallbladder. The right pleural cavity and infrahepatic space were also drained and lavaged with fluconazole.

explanation is that the cholecystitis became established in the gallbladder that had already been colonized by Candida albicans, with further growth of the fungus, following the infusion of the ERC contrast material, which was viscid and contained antibiotics. To our knowledge, this would be the first case in which ERC was a major contributing factor to Candida cholecystitis. Since the widespread use of ERC has increased the risk of exposing immunocompromised patients to this procedure, the development of Candida cholecystitis should be considered as a possible complication. Diabetes mellitus, even when well controlled, can also be a predisposing factor.

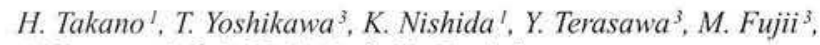
A. Yamaguchi ${ }^{2}$, B. Nishioka ${ }^{2}$, M. Kondo ${ }^{3}$

' Dept. of Medicine

${ }^{2}$ Dept. of Surgery, Shakaihoken Kobe Central Hospital, Kobe, Japan

${ }^{3}$ First Dept. of Medicine, Kyoto Prefectural University of Medicine, Kyoto, Japan

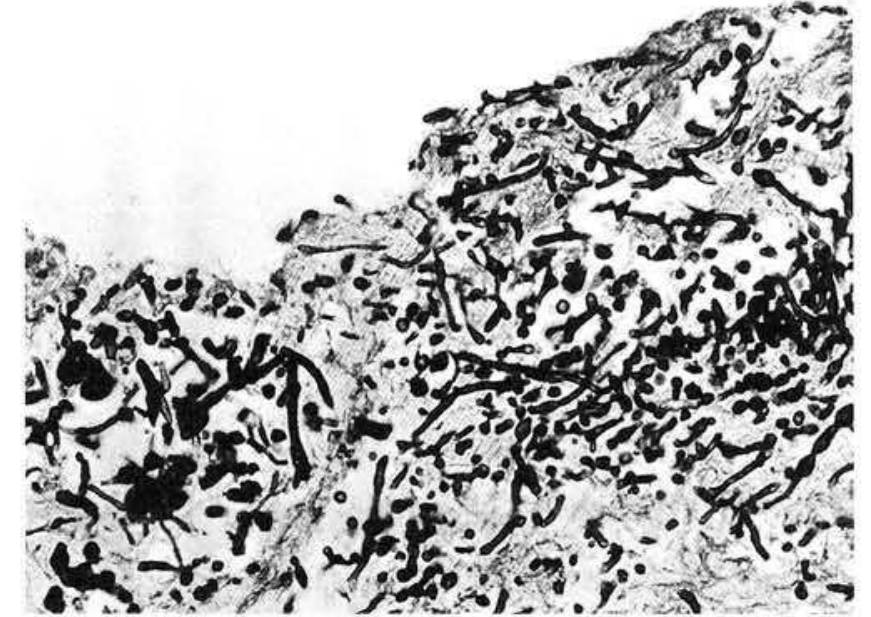

Figure 2: The histological specimen of the gallbladder shows the growth of Candida albicans even after systemic and local administration of fluconazole (periodic acid-Schiff stain, $\times 400$ ).

\section{References}

1. Haron E, Feld R, Tuffnell P, et al. Hepatic candidiasis: an increasing problem in immunocompromised patients. Am J Med 1987; 83: 17-26.

2. Hughes WT. Systemic candidiasis: a study of 109 fatal cases. Pediatr Infect Dis 1982; 1: 11-8.

3. Gips M, Halpern M, Wolloch Y. Acalculous Candida cholecystitis: case report. Eur J Surg 1992; 158: 251-2

4. Gomez-Mateos JM, Porto AS, Parra DM, et al. Disseminated candidiasis and gangrenous cholecystitis due to Candida spp. .J Infect Dis 1988; 158: 653-4.

5. Morris AB, Sands ML, Shiraki M, et al. Gallbladder and biliary tract candidiasis: nine cases and review. Rev Infect Dis 1990; 12: 483-9.

Corresponding Author

H. Takano, M.D.

National Institute for Environmental Studies

16-2 Onogawa

Tsukuba

Ibaraki 305

Japan

Fax: $+81-298-51-4732$ 